\title{
Combinatorial Interpretation of Raney Numbers and Tree Enumerations
}

\author{
Chin Hee Pah1, Mohamed Ridza Wahiddin² \\ ${ }^{1}$ Department of Computational and Theoretical Sciences, Faculty of Science, International Islamic University \\ Malaysia, Kuantan, Malaysia \\ ${ }^{2}$ Department of Computer Science, Faculty of ICT, International Islamic University Malaysia, Kuala Lumpur, \\ Malaysia \\ Email: pahchinhee@iium.edu.my, mridza@iium.edu.my
}

Received 24 November 2014; revised 20 December 2014; accepted 3 January 2015

Copyright (C) 2015 by authors and Scientific Research Publishing Inc.

This work is licensed under the Creative Commons Attribution International License (CC BY).

http://creativecommons.org/licenses/by/4.0/

\section{(c) (i) Open Access}

\section{Abstract}

A new combinatorial interpretation of Raney numbers is proposed. We apply this combinatorial interpretation to solve several tree enumeration counting problems. Further a generalized Catalan triangle is introduced and some of its properties are proved.

\section{Keywords}

Raney Numbers, Fuss-Catalan Numbers, Tree Enumeration, Network

\section{Introduction}

Interestingly Penson and Zyczkowski were the first who use the term Raney numbers [1] [2] and it is defined as $R_{k}(n, r)=\frac{r}{k n+r}\left(\begin{array}{c}k n+r \\ n\end{array}\right)$ where $k \geq 2, n \geq 1, r \geq 1$. Nevertheless, it is known that Raney's lemma could be used in counting problem associated with Catalan numbers [3] and a bijection exists between Raney path and plan multitree [4].

These numbers do not form novel sequences, as the numbers were introduced earlier as a generalization of the binomial series [5]. Moreover, the sequence

$$
R_{4}(n, 5)=1,5,30,200,1425,10626,81900,647280, \cdots
$$

is not included in OEIS database [6] before 2011. If we let $r=1$, we obtain another known sequence, i.e., Fuss-Catalan numbers [7] [8] which is defined as $C_{k}(n)=\frac{1}{(k-1) n+1}\left(\begin{array}{c}k n \\ n\end{array}\right)$. Although Fuss-Catalan numbers 
were introduced earlier than Catalan numbers [9], the Catalan numbers are more popular and widely used than the Fuss-Catalan numbers (see [10] [11] for details). Due to its self similar structure, the applications of Catalan numbers could be found in many physical problems, e.g., lattice model [12], tree enumeration network [13], and Hankel matrices in coding theory [14]. A tree is a connected graph with no cycles and for which only one shortest path exists from one node to another. Tree enumeration is an important tool to study network. These networks always grow in a power-law behavior which is often found in social network, subway system [15], etc.

In this paper, we introduce Raney numbers $R(k, r)$ in the form of a non-linear recursion and then we provide a combinatorial interpretation of Raney numbers. Using this combinatorial interpretation, we solve several tree enumeration counting problems in which we recover the well-known Fuss-Catalan numbers [16], Catalan triangles [17], and other less known numbers. Motivated by the connection between Raney numbers and Catalan triangles, a generalization of Catalan triangles is proposed and we prove some of their properties. Consequently these formulas generalize the properties of Catalan triangles. From the exact solution of these tree enumeration problems, we are able to find a sharp upper bound of the number of each tree enumeration problem. The upper bound is important in the contour method for lattice models and limit of the random graph.

\section{Raney Numbers}

Let $C_{k}(n)$ be the number of a $k$-ary trees with labeled $n$ vertices (Figure 1), where

$$
C_{k}(n)=\frac{1}{(k-1) n+1}\left(\begin{array}{c}
k n \\
n
\end{array}\right), \quad k \geq 2, n \geq 1 .
$$

The Raney numbers are defined as follows:

$$
R_{k}(n, r)=\sum_{i_{1}+i_{2}+\cdots+i_{r}=n} C_{k}\left(i_{1}\right) C_{k}\left(i_{2}\right) \cdots C_{k}\left(i_{r}\right), \quad C_{k}(0)=1, \quad \forall n>0
$$

where $i_{1}, i_{2}, \cdots, i_{r} \in \mathbb{N} \cup\{0\}$. Therefore, the combinatorial interpretation is as follows: $r$ copies of $k$-ary tree with total number of $n$ vertices.

Next, we let $u_{k}(x)$ be the generating function for $C_{k}(n)$, i.e.,

$$
u_{k}(x)=1+C_{k}(1) x+C_{k}(2) x^{2}+\cdots+C_{k}(n) x^{n}+\cdots .
$$

Then, the generating function of $R_{k}(n, r)$ is $u_{k}^{r}(x)$ and the Raney numbers satisfy the following formula [18].

Lemma 1. Let $u_{k}^{r}(x)$ be the generating function of the Raney numbers. Then,

$$
\left[x^{n}\right]\left(u^{r}(x)\right)=\frac{r}{k n+r}\left(\begin{array}{c}
k n+r \\
n
\end{array}\right) .
$$

Immediately, we obtain the following theorem.

Theorem 1. The binomial forms of the Raney numbers are given by

$$
R_{k}(n, r)=\frac{r}{k n+r}\left(\begin{array}{c}
k n+r \\
n
\end{array}\right) \text {. }
$$

From theorem (1), it is not difficult to deduce some of the properties of Raney numbers.

Corollary 1. For integer $k>1$, we have

$$
R_{k}(0, r)=1
$$
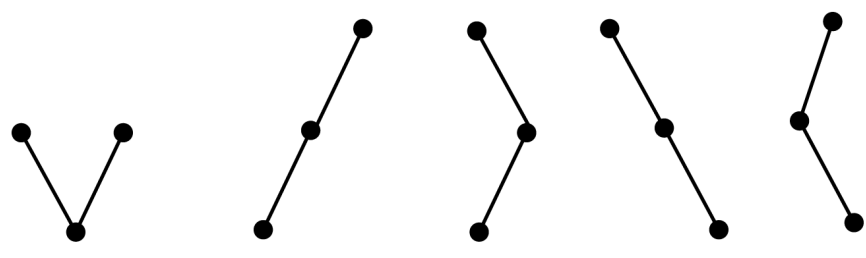

Figure 1. A binary tree with 3 nodes, where the bottom vertex is the root. 


$$
\begin{aligned}
& R_{k}(n-i, k i)=\frac{i}{n}\left(\begin{array}{c}
k n \\
n-i
\end{array}\right) ; \\
& R_{k}(n-1, k)=R_{k}(n, 1)=\frac{1}{(k-1) n+1}\left(\begin{array}{c}
k n \\
n
\end{array}\right) ; \\
& R_{k}(n, n)=\left(\begin{array}{c}
k n+n-1 \\
n-1
\end{array}\right) .
\end{aligned}
$$

Corollary 2. We can write $C_{k}(n)$ in a nonlinear recursion as:

$$
C_{k}(n+1)=\sum_{i_{1}+i_{2}+\cdots+i_{k}=n} C_{k}\left(i_{1}\right) C_{k}\left(i_{2}\right) \cdots C_{k}\left(i_{k}\right), \quad C_{k}(0)=1
$$

where $i_{1}, i_{2}, \cdots, i_{k} \in \mathbb{N} \bigcup\{0\}$.

We recover the formula by joining the $k$ copies of $k$-ary tree with $n$ vertices which is also equivalent to a $k$-ary tree with $n$ vertices and an additional root (see Figure 2):

$$
R_{k}(n, k)=R_{k}(n+1,1) .
$$

Using binomial form of $R_{k}(n, r)$, one can obtain the following result.

Corollary 3. For a fixed integer $k>1$, and $n \geq r>1$,

$$
R_{k}(n-1, r+k-1)=R_{k}(n, r)-R_{k}(n, r-1)
$$

where $R_{k}(n, r)=0$ if $n<r$.

For $k=2$, we recover the identity of a generalized Ballot numbers:

$$
R_{2}(n-1, r+1)=R_{2}(n, r)-R_{2}(n, r-1) .
$$

\section{A Homogeneous $k$-Ary Tree}

Unlike the usual $k$-ary tree, we define a homogeneous $k$-ary tree as a graph with no cycles, in which each vertex emanates $k+1$ edges (see Figure 3 for $k=4$ ). We fix a vertex namely $z$ as the root. Unlike the ordinary root in a $k$-ary tree, this root has $k+1$ successors while other vertices have $k$ number of successors. Any vertex could be chosen to be the root since the graph is homogenous. For a given $n$ vertices, we may find how many connected sub-tree rooted at $z$. This number is defined as $D_{k}(n)$.

Theorem 2. For $k>1$, we can write $D_{k}(n)$ in a nonlinear recursion of $C_{k}(n)$ as:

$$
D_{k}(n)=\sum_{r=1}^{n} C_{k}(r) C_{k}(n-r) \text { for } n>0 .
$$

Proof. We decompose the problem by finding out the number of $k$-ary tree of $n$ number of one copy of $k$-ary tree with $r$ vertices, i.e., $C_{k}(r)$, and another copy of $k$-ary tree with $n-r$ vertices, i.e. $C_{k}(n-r)$.

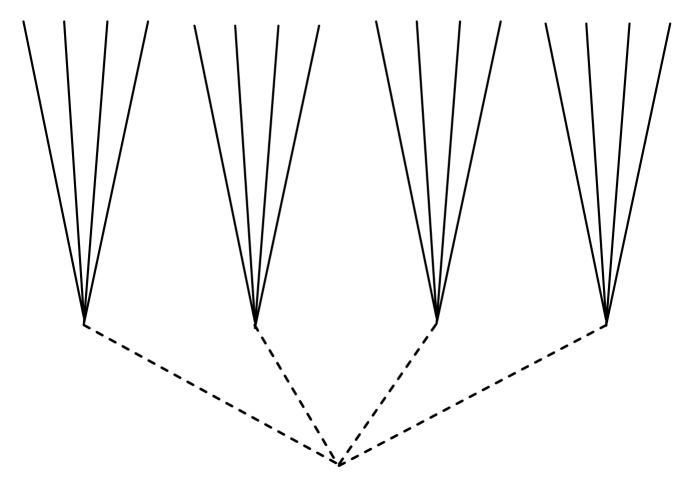

Figure 2. Joining 4 rooted Cayley tree of order 4 where $r=4$ and $k=4$. 


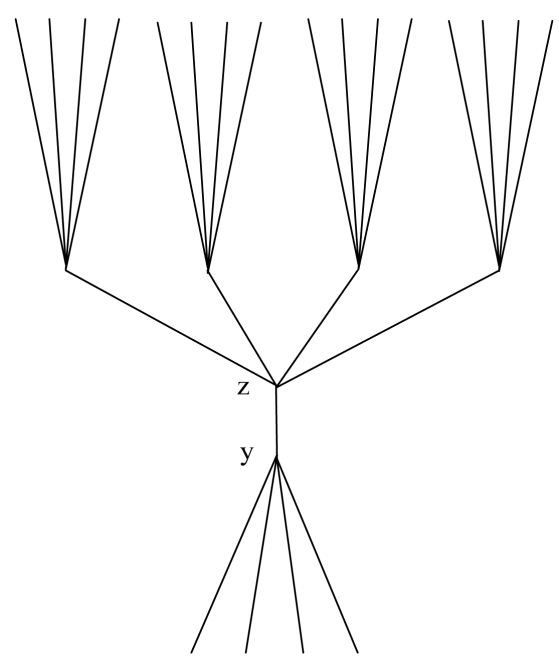

Figure 3. A homogenous graph where each vertex is connected to exactly 5 neighbours.

Since the former $C_{r}$ must always include $x^{0}$, its range should be from 1 to $n$. Total $D_{k}(n)$ is just the sum of all $C_{k}(r) C_{k}(n-r)$ using the addition and multiplication principles.

Using Equation (8), we rewrite the formula above as

$$
D_{k}(n)=\sum_{r_{1}+r_{2}+\cdots+r_{k+1}=n-1}^{n} C_{k}\left(r_{1}\right) C_{k}\left(r_{2}\right) \cdots C_{k}\left(r_{k+1}\right) \text { for } n>0 .
$$

This formula can also be obtained using $k+1$ copies of $k$-ary tree together with $n-1$ vertices and one center.

We then find the binomial form of $D_{k}(n)$.

Corollary 4. For $k \geq 2, D_{k}(n)$ is expressed in binomial form as:

$$
D_{k}(n)=R_{k}(n-1, k+1)=\frac{k+1}{(k-1) n+2}\left(\begin{array}{c}
k n \\
n-1
\end{array}\right) \quad \forall n>0 .
$$

Thus, $D_{2}(n)=\frac{3}{n+2}\left(\begin{array}{c}2 n \\ n-1\end{array}\right)$ and $D_{3}(n)=\frac{2}{n+2}\left(\begin{array}{c}3 n \\ n-1\end{array}\right)$ as in [19] and [20], respectively. For $k=4, D_{4}(n)$ coincides with one form of Raney numbers as mentioned above, i.e., $R_{4}(n, 5)$. The numbers $D_{k}(n)$ generate a lot of new sequences. For example, the sequence of $R_{5}(n, 6)$, i.e.,

$$
1,6,45,380,3450,32886,324632,3290040,34034715,357919100, \cdots
$$

is not found in the OEIS database [6].

From theorem (2), one can get

$$
D_{k}(n)=\sum_{r=0}^{n} C_{k}(r) C_{k}(n-r)-C_{k}(n) .
$$

This formula can be also obtained easily by a different way:

1) Count the number of trees by joining the 2 copies of $k$-nary tree, with total number of vertices $n$.

2) Subtract those trees remunerate from $y$ but doesn't contain $z$, that is, exactly the number $C_{k}(n)$.

Let the generating function of $D_{k}(n)$ be $w_{k}(x)$. Then we have the following result.

Corollary 5. For $k>1$ and $n \geq 0$, the generating function, $w_{k}(x)$ is

$$
w_{k}(x)=u_{k}^{2}(x)-u_{k}(x)
$$

where $u_{k}(x)$ is the generating function of $C_{k}(n)$.

Corollary 6. For $k>1$, 


$$
\sum_{r=1}^{n} C_{k}(r) C_{k}(n-r)=R_{k}(n-1, k+1)
$$

or

$$
\sum_{r=1}^{n} R_{k}(r, 1) R_{k}(n-r, 1)=R_{k}(n-1, k+1) .
$$

Using the binomial inequality in [21] and the binomial forms of $C_{k}(n)$ and $D_{k}(n)$, the following inequality can be easily proved.

Corollary 7. For $k>2$,

$$
C_{k}(n) \leq D_{k}(n)<\frac{\left(k \cdot b_{k}\right)^{n}}{n^{3 / 2}}
$$

where $b_{k}=\left(\frac{k}{k-1}\right)^{k-1}$ and $n>0$.

For sufficiently large $k$, a simpler form is produced as well, i.e., $C_{k}(n) \leq D_{k}(n)<\frac{(k \cdot e)^{n}}{n^{3 / 2}}$. These results are conjectured in a weaker form in [20], i.e., $C_{k}(n) \leq D_{k}(n)<\frac{(k \cdot e)^{n}}{n}$.

\section{Catalan Triangle}

A Catalan triangle $B(n, r)$ is defined as follows [9]:

$$
B(n, r)=\left\{\begin{array}{lc}
1 & \text { if } n=r=1 \\
B(n-1, r-1)+2 B(n-1, r)+B(n-1, r+1) & \text { if } 1 \leq r \leq n \\
0 & \text { otherwise }
\end{array}\right.
$$

The Catalan triangle satisfies [9]:

$$
B(n, r)=\sum_{\substack{i_{1}+\cdots+i_{r}=n \\ i_{1}, \cdots, i_{r}=1}} C_{2}\left(i_{1}\right) C_{2}\left(i_{2}\right) \cdots C_{2}\left(i_{r}\right), \quad C_{2}(0)=1 \quad \forall n>0
$$

Using a property of Catalan numbers, $C_{2}\left(i_{r}\right)=\sum_{j_{1}+j_{2}=i_{r}-1} C_{2}\left(j_{1}\right) C_{2}\left(j_{2}\right)$, where $j_{1}, j_{2} \geq 0$, we get another form of $B(n, r)$, i.e.,

$$
B(n, r)=\sum_{j_{1}+j_{2}+\cdots+j_{r}=n-r} C_{2}\left(j_{1}\right) C_{2}\left(j_{2}\right) \cdots C_{2}\left(j_{2 r}\right),
$$

where $j_{1}, j_{2}, \cdots, j_{2 r} \in \mathbb{N} \bigcup\{0\}$. From Equation (1), we immediately recover the Catalan triangle from the Raney numbers, i.e., $B(n, r)=R_{2}(n-r, 2 r)$ :

$$
B(n, r)=R_{2}(n-r, 2 r)=\frac{r}{n}\left(\begin{array}{c}
2 n \\
n-r
\end{array}\right) .
$$

We now consider the following problem as in [22]: Find out the number of all different connected sub-trees of a homogenous binary tree with $n$ number of vertices, containing the given $r$ number of fixed vertices (where $n \geq 2 r-2$ ). The condition, $n \geq 2 r-2$, is simply the number of vertices that covers the minimal component containing all $r$ vertices. The details of this problem and terminologies could be found in the original paper [22]. We denote the solution to this problem as $F_{n}^{r}$. In this paper, we show that a solution to the case when the minimal component is "full", is as below:

$$
F_{n}^{r}=B(n-r+2, r)=\frac{r}{n-r+2}\left(\begin{array}{c}
2(n-r+2) \\
n+2
\end{array}\right),
$$


where $n$ is the number of given vertices and $r$ is the number of fixed vertices in each of the connected subtree.

Now, we interpret and relate the problem above with the combinatorial interpretation of the Raney numbers through the following steps (see Figure 4):

1) Given $n$ vertices;

2) Fill up all the interior points, i.e., $r-2$;

3) Fill up all the boundary points, i.e., $r$;

4) Then only $n-2 r+2$ vertices are left;

5) Since each boundary point has 2 neighbours which is not an interior point, we have $2 r$ boxes;

6) If $n-2 r+2$ vertices are given, then there are $2 r$ boxes of binary tree to be filled.

As a result, the solution is

$$
R_{2}(n-2 r+2,2 r)=\frac{2 r}{2(n-2 r+2)+2 r}\left(\begin{array}{c}
2(n-2 r+2)+2 r \\
n-2 r+2
\end{array}\right)=\frac{r}{n-r+2}\left(\begin{array}{c}
2(n-r+2) \\
n+2
\end{array}\right)
$$

Furthermore, it is natural to define a generalized Catalan triangle, i.e., $k$-th Catalan triangle using FussCatalan numbers instead of Catalan numbers as in Equation (19):

$$
B_{k}(n, r)=\sum_{\substack{i_{1}+\cdots+i_{r}=n \\ i_{1}, \cdots, i_{r} \geq 1}} C_{k}\left(i_{1}\right) C_{k}\left(i_{2}\right) \cdots C_{k}\left(i_{r}\right), \quad C_{k}(0)=1 \quad \forall n>0,
$$

where $B_{k}(n, r)=0$ if $n<r$.

From the property of Fuss-Catalan numbers, i.e., corollary (2)

$$
C_{k}\left(i_{r}\right)=\sum_{j_{1}+j_{2}+\cdots+j_{k}=i_{r}-1} C_{k}\left(j_{1}\right) C_{k}\left(j_{2}\right) \cdots C_{k}\left(j_{k}\right),
$$

where $j_{1}, j_{2}, \cdots, j_{k} \geq 0$, we find another form of $B_{k}(n, r)$,

$$
B_{k}(n, r)=\sum_{j_{1}+j_{2}+\cdots+j_{r}=n-r} C_{k}\left(j_{1}\right) C_{k}\left(j_{2}\right) \cdots C_{k}\left(j_{k r}\right),
$$

where $j_{1}, j_{2}, \cdots, j_{k r} \in \mathbb{N} \bigcup\{0\}$. Again, from Equation (1), we immediately have

$$
B_{k}(n, r)=R_{k}(n-r, k r)=\frac{r}{n}\left(\begin{array}{c}
k n \\
n-r
\end{array}\right) .
$$

Lemma 2. Some properties of $k$-th Catalan triangles are as follows:

$$
\begin{aligned}
& B_{k}(n, 1)=C_{k}(n), \\
& B_{k}(n, n)=1, \\
& B_{k}(n, n-1)=(n-1) k .
\end{aligned}
$$

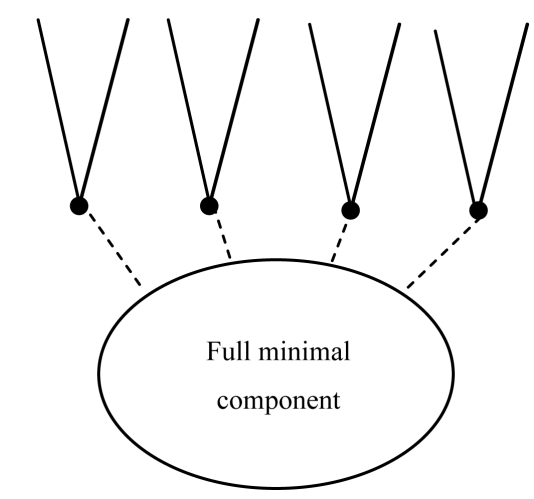

Figure 4. 4 boundary points (solid circles) connected to full minimal component. 
Using the binomial form of $B_{k}(n, r)$, one can show that:

Lemma 3. For $k>1$ and $n \geq r \geq 2$

$$
B_{k}(n-1, r-1)+2 B_{k}(n-1, r)+B_{k}(n-1, r+1)=\frac{k r-k+2}{k n-k+2}\left(\begin{array}{c}
k n-k+2 \\
n-r
\end{array}\right) \text {. }
$$

If $k=2$, we recover

$$
B_{2}(n-1, r-1)+2 B_{2}(n-1, r)+B_{2}(n-1, r+1)=\frac{r}{n}\left(\begin{array}{c}
2 n \\
n-r
\end{array}\right)=B_{2}(n, r) .
$$

Based on the initial result, lemma (3), we prove the following assertion by mathematical induction with respect to $m$.

Theorem 3. For fixed $k$, where $k \geq m>1$, and $n \geq r>1$,

$$
\sum_{i=0}^{m}\left(\begin{array}{c}
m \\
i
\end{array}\right) B_{k}(n-1, r-1+i)=\frac{k r-k+m}{k n-k+m}\left(\begin{array}{c}
k n-k+m \\
n-r
\end{array}\right),
$$

where $B_{k}(n, r)=0$ if $n<r$.

Proof. Assertion is true for $m=2$. Assume that it is true for $m$, we consider the following summations:

$$
\begin{aligned}
& \sum_{i=0}^{m}\left(\begin{array}{c}
m \\
i
\end{array}\right) B_{k}(n-1, r-1+i)+\sum_{i=0}^{m}\left(\begin{array}{c}
m \\
i
\end{array}\right) B_{k}(n-1, r+i) \\
& =\frac{k r-k+m}{k n-k+m}\left(\begin{array}{c}
k n-k+m \\
n-r
\end{array}\right)+\frac{k(r+1)-k+m}{k n-k+m}\left(\begin{array}{c}
k n-k+m \\
n-r-1
\end{array}\right) . \\
& B_{k}(n-1, r-1)+\sum_{i=1}^{m}\left(\begin{array}{c}
m \\
i
\end{array}\right) B_{k}(n-1, r-1+i)+\sum_{i=0}^{m-1}\left(\begin{array}{c}
m \\
i
\end{array}\right) B_{k}(n-1, r+i)+B_{k}(n-1, r+m) \\
& =\frac{k r-k+(m+1)}{k n-k+(m+1)}\left(\begin{array}{c}
k n-k+m+1 \\
n-r
\end{array}\right) \text {. } \\
& B_{k}(n-1, r-1)+\sum_{i=1}^{m}\left(\left(\begin{array}{c}
m \\
i
\end{array}\right)+\left(\begin{array}{c}
m \\
i-1
\end{array}\right)\right) B_{k}(n-1, r-1+i)+B_{k}(n-1, r+m) \\
& =\frac{k r-k+(m+1)}{k n-k+(m+1)}\left(\begin{array}{c}
k n-k+m+1 \\
n-r
\end{array}\right) \text {. } \\
& B_{k}(n-1, r-1)+\sum_{i=1}^{m}\left(\begin{array}{c}
m+1 \\
i
\end{array}\right) B_{k}(n-1, r-1+i)+B_{k}(n-1, r+m) \\
& =\frac{k r-k+(m+1)}{k n-k+(m+1)}\left(\begin{array}{c}
k n-k+m+1 \\
n-r
\end{array}\right) \text {. } \\
& \sum_{i=0}^{m+1}\left(\begin{array}{c}
m+1 \\
i
\end{array}\right) B_{k}(n-1, r-1+i)=\frac{k r-k+(m+1)}{k n-k+(m+1)}\left(\begin{array}{c}
k n-k+m+1 \\
n-r
\end{array}\right) \text {. }
\end{aligned}
$$

Hence, the assertion is true for any $m>1$.

Corollary 8. For fixed $k$, where $k \geq m>1$, and $n \geq r>1$, we have

$$
\sum_{i=0}^{m} \frac{r+i}{n}\left(\begin{array}{c}
m \\
i
\end{array}\right)\left(\begin{array}{c}
k n \\
n-r-i
\end{array}\right)=\frac{k r+m}{k n+m}\left(\begin{array}{c}
k n+m \\
n-r
\end{array}\right) \text {. }
$$

For $m=k$, we have the following simple result:

Corollary 9. For fixed $k>1$, and $n \geq r>1$,

$$
B_{k}(n, r)=\sum_{i=0}^{k}\left(\begin{array}{l}
k \\
i
\end{array}\right) B_{k}(n-1, r-1+i) \text {, }
$$


where $B_{k}(n, r)=0$ if $n<r$.

\section{Binomial Transformation of $\boldsymbol{k}$-th Catalan Triangle}

For $r \geq 2$ and $n \geq r$, we define a new number $H_{k, m}^{(r)}(n)$ as:

$$
H_{k, m}^{(r)}(n)=\sum_{\substack{j_{1}+\cdots+j_{m}+l_{1}+\cdots+l_{l}=n \\ j_{1}, \cdots, j_{m} \geq 0, k_{1}, \cdots, l_{r} \geq 1}} C_{k}\left(j_{1}\right) \cdots C_{k}\left(j_{m}\right) \cdot C_{k}\left(l_{1}\right) \cdots C_{k}\left(l_{r}\right) .
$$

If $j_{1}=j_{2}=\cdots=j_{m}=0$, all the Fuss-Catalan numbers should start at 1 , then we recover the previously defined $k$-th Catalan triangle.

From the property of Fuss-Catalan numbers, $C_{k}\left(i_{r}\right)=\sum_{j_{1}+j_{2}+\cdots+j_{k}=i_{r}-1} C_{k}\left(j_{1}\right) C_{k}\left(j_{2}\right) \cdots C_{k}\left(j_{k}\right)$, where $j_{1}, j_{2}, \cdots \geq 0$, we found another form of

$$
H_{k, m}^{(r)}(n)=\sum_{\substack{j_{1}+\cdots+j_{m}+l_{1}+\cdots+l_{l}=n-r \\ j_{1}, \cdots, j_{r}, l_{1}, \cdots, l_{k r} \geq 0}} C_{k}\left(j_{1}\right) \cdots C_{k}\left(j_{m}\right) \cdot C_{k}\left(l_{1}\right) \cdots C_{k}\left(l_{k r}\right) .
$$

From Equation (1), we immediately have

$$
H_{k, m}^{(r)}(n)=H_{k, m+k r}^{(0)}(n-r)=R_{k}(n-r, m+k r)=\frac{m+k r}{k n+m}\left(\begin{array}{c}
k n+m \\
n-r
\end{array}\right)
$$

and for $m=0$, we recover the same formula for $k$-th Catalan triangle,

$$
H_{k, m}^{(r)}=R_{k}(n-r, k r)=\frac{r}{n}\left(\begin{array}{c}
k n \\
n-r
\end{array}\right) .
$$

From theorem (3), $H_{k, m}^{(r)}(n)$ is obtained as a result of binomial transformation of $k$-Catalan triangles.

Corollary 10. For fixed $k>1$, where $k \geq m>1$, and $n \geq r>1$,

$$
\sum_{i=0}^{m}\left(\begin{array}{c}
m \\
i
\end{array}\right) B_{k}(n, r+i)=H_{k, m}^{(r)}(n)
$$

where $B_{k}(n, r)=0$ if $n<r$.

\section{Conclusion}

In this paper, we have introduced the combinatorial interpretation of Raney numbers to solve various tree enumeration counting problems. The upper bound of any $k+1$ order tree enumeration is generally found to be $\frac{(k \cdot e)^{n}}{n^{3 / 2}}$. We have also shown how a new number $H_{k, m}^{(r)}(n)$ may be derived from the binomial transformation of $k$-th Catalan triangles.

\section{Acknowledgements}

This research is funded by the MOHE grant FRGS11-022-0170. The authors are grateful to anoymous referee's suggestion and improvement of the presentation of this paper.

\section{References}

[1] Penson, K.A. and Zyczkowski, K. (2011) Product of Ginibre Matrices: Fuss-Catalan and Raney Distributions. Physical Review E, 83, Article ID: 061118. http://dx.doi.org/10.1103/PhysRevE.83.061118

[2] Mlotkowski, W., Penson, K.A. and Zyczkowski, K. (2013) Densities of the Raney Distributions. Documenta Mathematica, 18, 1573-1596.

[3] Jeurissen, R.H. (2008) Raney and Catalan. Discrete Mathematics, 308, 6298-6307. http://dx.doi.org/10.1016/j.disc.2007.11.068

[4] Dziemiaczuk, M. (2014) Enumerations of Plane Trees with Multiple Edges and Raney Lattice Paths. Discrete Mathe- 
matics, 337, 9-24. http://dx.doi.org/10.1016/j.disc.2014.07.024

[5] Gould, H.W. (1972) Combinatorial Identities: A Standardized Set of Tables Listing 500 Binomial Coefficient Summations. Morgantown.

[6] (2011) The On-Line Encyclopedia of Integer Sequences. Sequence A196678. http://oeis.org

[7] Graham, R.L., Knuth, D.E. and Patashnik, O. (1994) Concrete Mathematics. Addison-Wesley, Boston.

[8] Hilton, P. and Pedersen, J. (1991) Catalan Numbers, Their Generalization, and Their Uses. Mathematical Intelligencer, 13, 64-75. http://dx.doi.org/10.1007/BF03024089

[9] Koshy, T. (2008) Catalan Numbers with Applications. Oxford University Press, USA. http://dx.doi.org/10.1093/acprof:oso/9780195334548.001.0001

[10] Stanley, R.P. (2013) Catalan Addendum to Enumerative Combinatorics. Vol. 2.

[11] Stanley, R.P. (1999) Enumerative Combinatorics. Vol. 2, Cambridge University Press, Cambridge. http://dx.doi.org/10.1017/CBO9780511609589

[12] Baxter, R.J. (1982) Exactly Solved Models in Statistical Mechanics. Academic Press, London.

[13] Goltsev, A.V., Dorogovtsev, S.N. and Mendes, J.F.F. (2008) Critical Phenomena in Complex Networks. Reviews of Modern Physics, 80, 1275. http://dx.doi.org/10.1103/RevModPhys.80.1275

[14] Tamm, U. (2001) Some Aspects of Hankel Matrices in Coding Theory and Combinatorics. The Electronic Journal of Combinatorics, 8, 1-31.

[15] Lee, K., Jung, W.S., Park, J.S. and Choi, M.Y. (2000) Statistical Analysis of the Metropolitan Seoul Subway System: Network Structure and Passenger Flows. Physica A, 387, 6231-6234. http://dx.doi.org/10.1016/j.physa.2008.06.035

[16] Aval, J.C. (2008) Multivariate Fuss-Catalan Numbers. Discrete Mathematics, 308, 4660-4669. http://dx.doi.org/10.1016/j.disc.2007.08.100

[17] Shapiro, L.W. (1976) A Catalan Triangle. Discrete Mathematics, 14, 83-90. http://dx.doi.org/10.1016/0012-365X(76)90009-1

[18] Merlini, D., Sprugnoli, R. and Verri, M.C. (2006) Lagrange Inversion: When and How. Acta Applicandae Mathematica, 94, 233-249. http://dx.doi.org/10.1007/s10440-006-9077-7

[19] Pah, C.H. (2008) An Application of Catalan Number on Cayley Tree of Order 2: Single Polygon Counting. Bulletin of the Malaysian Mathematical Sciences Society, 31, 175-183.

[20] Pah, C.H. (2010) Single Polygon Counting on Cayley Tree of Order 3. Journal of Statistical Physics, 140, 198-207. http://dx.doi.org/10.1007/s10955-010-9989-5

[21] Sasvari, Z. (1999) Inequalities for Binomial Coefficients. Journal of Mathematical Analysis and Applications, 236, 223-226. http://dx.doi.org/10.1006/jmaa.1999.6420

[22] Mukhomedov, F., Pah, C.H. and Saburov, M. (2010) Single Polygon Counting for $m$ Fixed Nodes in Cayley Tree: Two Extremal Cases. Preprint. http://arxiv.org/abs/1004.2305 
Scientific Research Publishing (SCIRP) is one of the largest Open Access journal publishers. It is currently publishing more than 200 open access, online, peer-reviewed journals covering a wide range of academic disciplines. SCIRP serves the worldwide academic communities and contributes to the progress and application of science with its publication.

Other selected journals from SCIRP are listed as below. Submit your manuscript to us via either submit@scirp.org or Online Submission Portal.
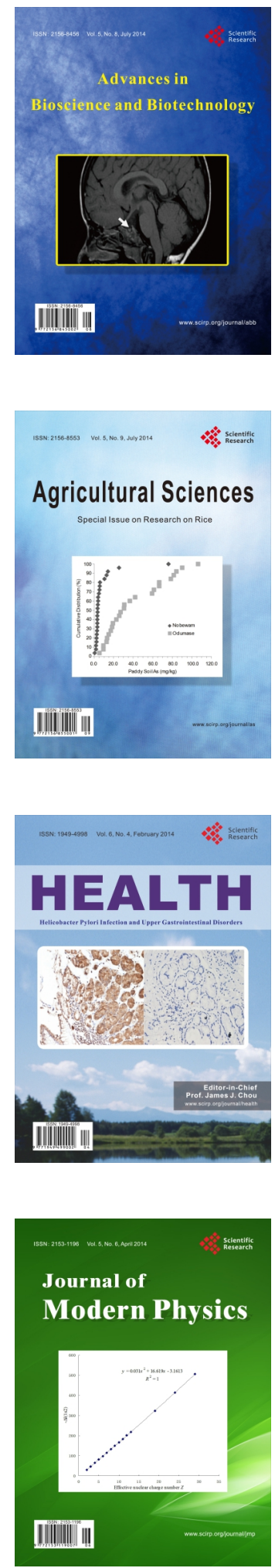
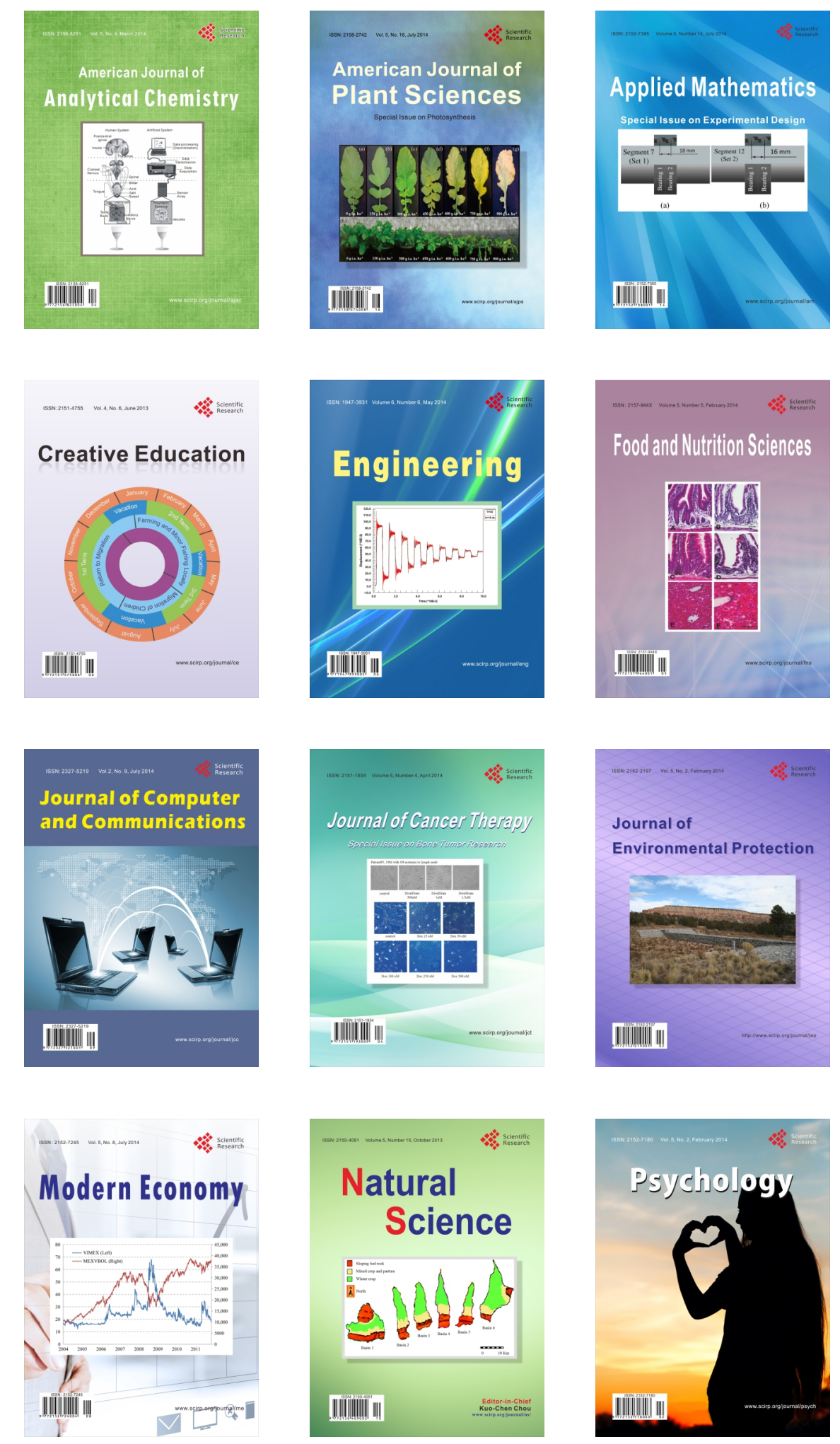